\title{
Contribuição para o conhecimento do gênero Arlesia Handschin (Collembola, Neanuridae, Pseudachorutinae)
}

\author{
Maria Cleide de Mendonça ${ }^{1}$ \\ Liliane Henriques Fernandes ${ }^{1}$
}

\begin{abstract}
Contribution to the knowledge of the genus Arlesia Handschin (Collembola, Neanuridae, Pseudachorutinae). Arlesia arleana sp.n. is described and illustrated. Arlesia proxima (Arlé, 1939) is redescribed based on especimens from Ubatuba, São Paulo, Brazil.

KEY WORDS. Collembola, Pseudachorutinae, Arlesia arleana, Arlesia proxima, taxonomy
\end{abstract}

O gênero Arlesia Handschin, 1942 foi proposto para abrigar as espécies Pseudachorutes albipes Folsom, 1927 (do Panamá), P. fluminensis Arlé, 1939 e $P$. proximus Arlé, 1939 (ambas do Brasil). Posteriormente, foram incluídas as espécies Arlesia cochabambensis Cassagnau \& Rapoport, 1962 (Bolívia), A. minima (Massoud, 1963) (Angola), A. delamarei (Murphy, 1965) (Gambia), A. microphtalma (Barra, 1969) (Gabão), A. pillaii Prabhoo, 1971 (Índia) e A. variabilis Thibaud \& Massoud, 1983 (Guadalupe). Dessas espécies, A. minima, A. delamarei e A. microphtalma foram transferidas para o gênero Cephalachorutes Bedos \& Deharveng, 1991. Segundo estes autores, A. pillaii poderia também ter sido incluída em Cephalachorutes, não fosse a descrição insuficiente da mesma.

ARLÉ \& RUFINO (1976) criaram Arlesia albipes f. decorata Arlé \& Rufino, 1976 para dois espécimens procedentes do Município Tiúma, Estado de Pernambuco. Nesse trabalho, os autores afirmaram que Arlesia albipes $f$. decorata era morfologicamente idêntica à $A$. albipes, diferindo pela coloração. Durante excursões realizadas à Reserva Florestal do Curado, Município de Recife, encontrou-se inúmeros exemplares em folhiço do chão de mata que correspondiam à Arlesia albipes $f$. decorata. Porém, o material por nós coletado apresentou diferenças significativas, especialmente as que se referem às peças bucais, que comprovam ser um táxon distinto dos demais incluídos no gênero. De acordo com o ICZN (1985: Art. 1b (5) e Art. 16) um nome proposto após 1960 como variedade ou forma é considerado infra-subespecífico e, como tal, não disponível e excluído da nomenclatura zoológica. Considera-se no presente trabalho Arlesia albipes $f$. decorata, eomo sendo uma espécie distinta a denominar-se $A$. arleana sp.n., aqui descrita e ilustrada. Acrescenta-se também a redescrição de A. proxima (Arlé, 1939) com o objetivo de fornecer informações adicionais à sua descrição original.

O material estudado está depositado na Coleção de Collembola, Departamento de Entomologia, Museu Nacional, Rio de Janeiro (CM/MNRJ).

1) Departamento de Entomologia, Museu Nacional. Quinta da Boa Vista, São Cristóvão, 20940-040 Rio de Janeiro, Rio de Janeiro, Brasil. 

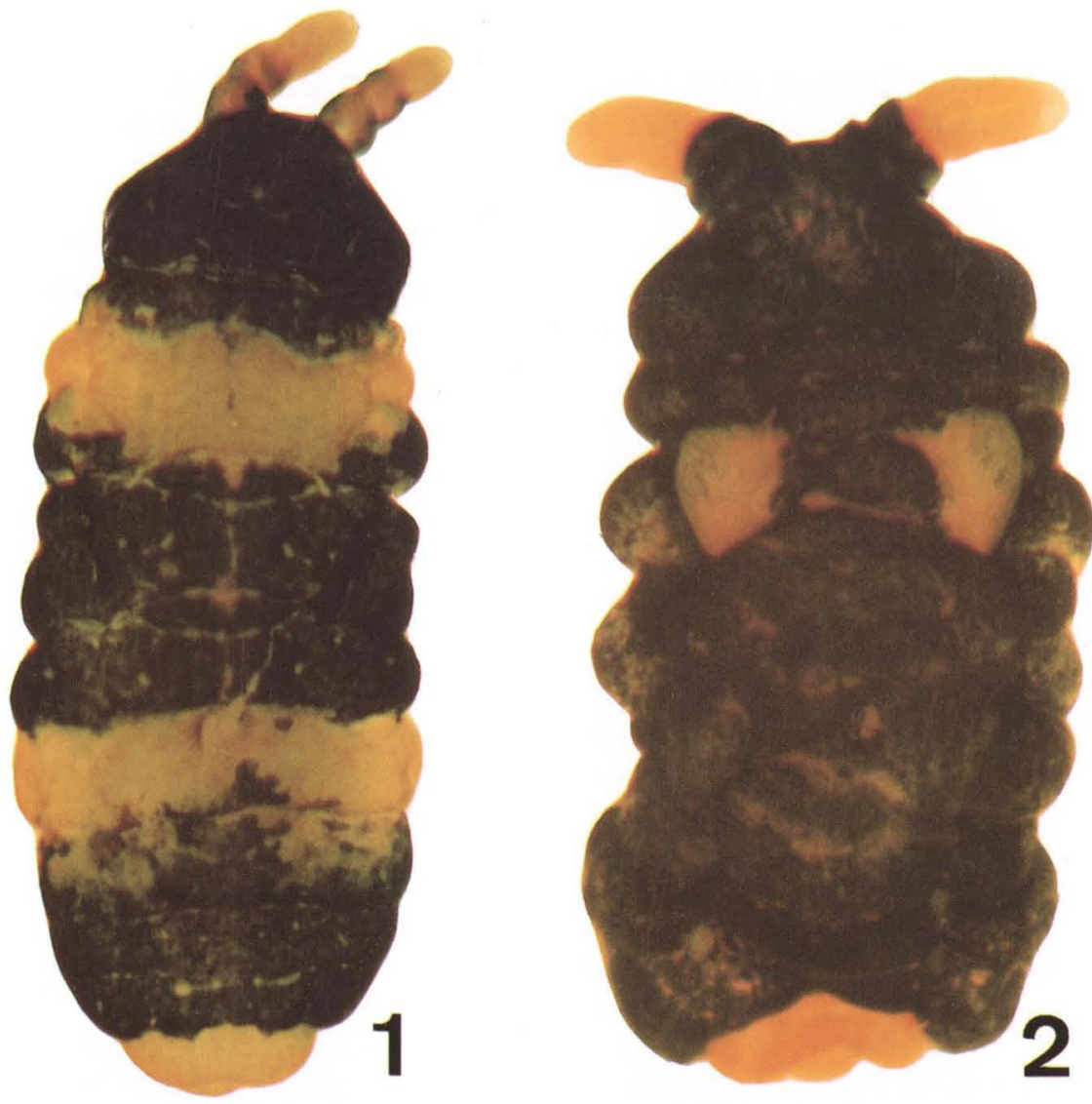

Figs 1-2. (1) Arlesia arleana sp.n., comprimento 2,24 mm; (2) Arlesia proxima (Arlé, 1939), comprimento $1,77 \mathrm{~mm}$.

\section{Arlesia arleana sp.n.}

Figs 1, 3-17

Arlesia albipes f. decorata Arlé \& Rufino, 1976: 106 [nom. nud., ICZN, Art. 1b (5), Art. 16].

Comprimento Holótipo $1,88 \mathrm{~mm}$; parátipos $0,91 \mathrm{~mm}$ a 2,24mm.

Coloração geral escura, quase preta, com faixas amareladas no tergito torácico II e tergitos abdominais II, V e VI; antenômeros I e II amarelados na parte central, e antenômeros III e IV totalmente amarelados (Fig. 1); pigmentação esbranquiçada na região central da face ventral dos segmentos torácicos e abdominais, nas patas e no cone bucal.

Tegumento fortemente granuloso. Habitus típico de Pseudachorutini, com paratergitos arredondados do tergito torácico I ao tergito abdominal II; tergito abdominal V não deformado e o VI visível dorsalmente. Quetotaxia formada por microquetas e cerdas sensoriais longas, lisas e arredondadas no ápice; fórmula sensorial por meio-tergito $=022 / 11111$ (Fig. 3) . 


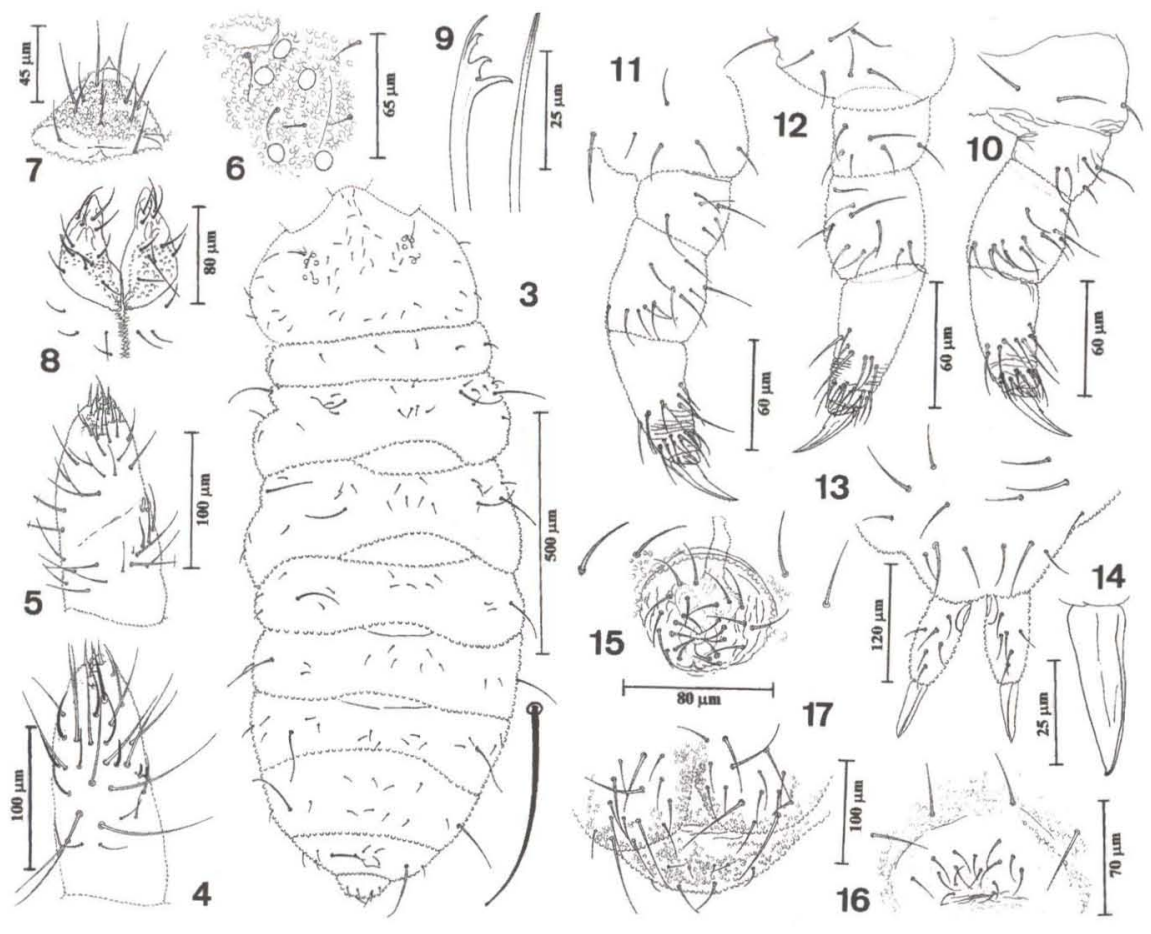

Figs 3-17. Arlesia arleana sp.n.. (3) Quetotaxia dorsal; (4) face dorso-lateral dos antenômeros III e IV; (5) face ventral dos antenômeros III e IV; (6) grupo ocular; (7) quetotaxia do labro; (8) cerdas labiais; (9) maxila e mandíbula; (10) Pata I; (11) pata II; (12) pata III; (13) furca; (14) vista lateral do mucro; (15) placa genital masculina; (16) placa genital feminina; (17) quetotaxia das valvas anais.

Antenas mais curtas que a diagonal cefálica; relação entre o comprimento das antenas e diagonal cefálica $=0,82: 1,0$; antenômeros I e II com 10 e 11 cerdas respectivamente; órgão sensorial do antenômero III constituído de dois túbulos retos separados, duas sensilas de guarda e pequena sensila ventral; antenômero IV com vesícula apical trilobada, uma micro sensila levemente côncava, alojada em fosseta, e sete sensilas subcilíndricas entre cerdas finas de tamanhos variados (Figs 4-5).

Cornéulas 5+5. Órgão pós-antenal ausente, havendo, em substituição, pequena área de granulação mais fina (Fig. 6). Cone bucal curto e afilado com a quetotaxia labral $=2 / 352$ (Fig. 7) e cerdas labiais conforme figura 8. Maxila estiliforme; mandíbula com 6 dentes, sendo o basal e o apical longos e finos, e os medianos curtos e providos de pequeno dente (Fig. 9).

Tibiotarsos I, II, e III com 19, 19, e 18 cerdas respectivamente; unha medindo aproximadamente $36 \mu \mathrm{m}$, com um dente interno, sem ergot distinto (Figs 10-12).

Tubo ventral com $3+3$ cerdas. Tenáculo com $3+3$ dentes.

Furca normalmente desenvolvida; manúbrio com 14 cerdas na face dorsal, e dens com seis cerdas subiguais (Fig. 13); mucro reto com extremidade ligeiramente curva (Fig. 14); relação do comprimento dens:mucro $=1,0: 0,70$. 


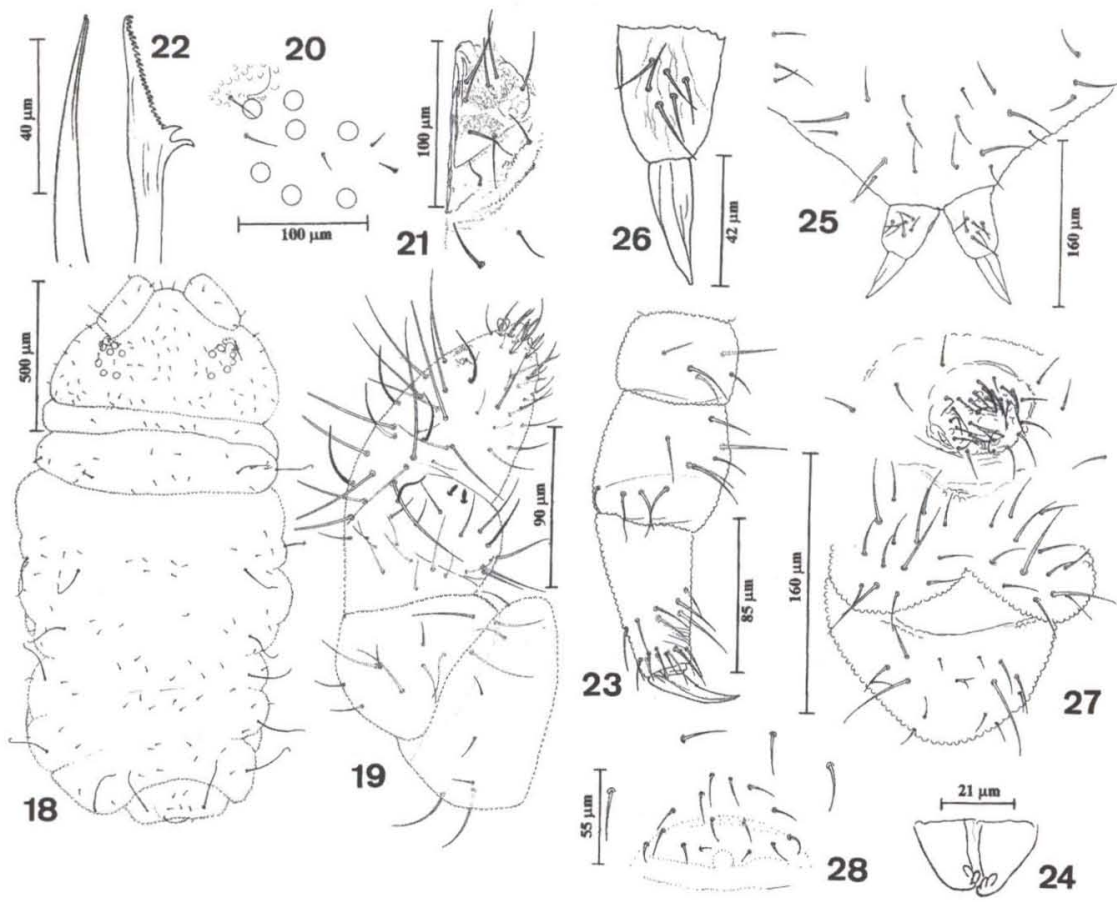

Figs 18-28. Arlesia proxima. (18) Quetotaxia dorsal; (19) quetotaxia da antena; (20) grupo ocular; (21) cerdas labiais; (22) maxila e mandibula; (23) Pata III; (24) retináculo; (25) furca; (26) dens e mucro; (27) placa genital do macho e quetotaxia das valvas anais; (28) placa genital feminina.

Placa genital com cerca de 22 cerdas dispostas em dois círculos no macho (Fig. 15) e cerca de 15 cerdas dispostas em semicírculo na fêmea (Fig. 16); aspecto ventral dos segmentos abdominais V e VI conforme figura 17.

Discussão. Comparando A. arleana com as demais espécies do gênero constatou-se semelhanças com A. albipes no tocante ao número de olhos, cerdas labiais, cerdas dentais e forma da unha e do mucro. Todavia, o exame minucioso da mandíbula de $A$. arleana revelou uma morfologia indiscutivelmente distinta da mandíbula de A. albipes ilustrada por DeNIS (1931), MAssoud $(1963,1967)$ e THIBAUD \& MASSOUD (1983). Verificou-se ainda que A. arleana apresenta as cerdas sensoriais longas e levemente arredondadas no ápice; o número de sensilas do antenômero IV, número de cerdas do tergito torácico I, número de microquetas centrais do tergito abdominal $\mathrm{I}$, e microquetas laterais dos tergitos abdominais II e III diferentes daquelas mostradas por MASSOUD (1963) para A. albipes. Além disso, A. arleana é provavelmente espécie endêmica da região nordeste com padrão de coloração fixo, desde os indivíduos jovens até os adultos. Ao passo que a espécie A. albipes amplamente distribuída desde o México até o Brasil, tem sido encontrada nos mais diversos ambientes, apresentando coloração acinzentada, amarronzada e azulada em diferentes graus de tonalidade. 
Quanto à A. arleana, acredita-se tratar-se de uma população simpátrica, porém distinta da de Arlesia albipes (Folsom). A localidade de Tiúma, onde foram coletados os exemplares de $A$. albipes $f$. decorata, é muito próxima de Recife, a localidade tipo de $A$. arleana. Provavelmente, devido ao número muito reduzido de exemplares examinados, ARLÉ \& RUFINO (1976) preferiram considerar as diferenças constatadas como simples variações intra-específicas.

Material examinado. Holótipo fêmea. BRAsIL, Pernambuco: Recife (Reserva Florestal do Curado, em lâmina 405 CM/MNRJ), 10-IV-1993, C. Mendonça leg. . Parátipos com a mesma procedência do holótipo: 1 jovem em lâmina 320 CM/MNRJ; 1 jovem em lâmina 321 CM/MNRJ, 03-IX-90, C. Mendonça leg.; 1 macho e 4 fêmeas em lâmina 405 CM/MNRJ, 10-IV-93, C. Mendonça leg.; 1 fêmea em lâmina 414 CM/MNRJ, 10-IV-93, C. Mendonça leg.; 1 fềmea em lâmina 418 CM/MNRJ, 10-IV-93, C. Mendonça leg.; 1 fềmea em lâmina 739 CM/MNRJ, 04-VIII-97, C. Mendonça leg.; 74 parátipos em álcool 70\% com as seguintes numerações: 319 CM/MNRJ, 03-IX-90, 401 CM/MNRJ, 403 CM/MNRJ, 404 $\mathrm{CM} / \mathrm{MNRJ}, 405 \mathrm{CM} / \mathrm{MNRJ}, 406 \mathrm{CM} / \mathrm{MNRJ}, 407 \mathrm{CM} / \mathrm{MNRJ}, 409 \mathrm{CM} / \mathrm{MNRJ}, 410$ CM/MNRJ, 411 CM/MNRJ, 412 CM/MNRJ, 414 CM/MNRJ, 415 CM/MNRJ, 417 CM/MNRJ, 418 CM/MNRJ, 10-IV-1993, C. Mendonça leg..

Etimologia. O nome espécifico arleana, refere-se à fusão dos nomes Arlé e Elisiana, em homenagem aos colegas Roger Arlé e Elisiana de Oliveira.

\section{Arlesia proxima (Arlé, 1939)}

Figs 2, 18-28

Pseudachorutes proximus Arlé, 1939: 69.

Arlesia proxima Handschin, 1942: 282. - Arlé \& Rufino, 1976: 106.

Handschinia proxima Stach, 1949: 57.

Comprimento médio $1,72 \mathrm{~mm}$.

Coloração conspícua com tegumento geral muito escuro, um par de manchas brancas ou alaranjadas mais ou menos redondas na face dorsal dos tergitos torácicos II e III; antenômeros III e IV e tergitos abdominais V e VI amarelo-alaranjados (Fig. 2); região central e ventral dos segmentos torácicos e abdominais, patas e cone bucal esbranquiçados. Tegumento fortemente granuloso. Habitus típico de Pseudachorutini (tipo 3) com paratergitos salientes; tergito abdominal VI pouco visível dorsalmente. Quetotaxia formada por microquetas e cerdas sensoriais longas, lisas e levemente arredondadas no ápice (Fig. 18); fórmula sensorial por meio-tergito $=022 / 11111$.

Antenas mais curtas que a diagonal cefálica; relação comprimento antena: cabeça $=0,65$ : 1,0; antenômeros I e II com 7 e 11 cerdas respectivamente; órgão sensorial do antenômero III formado por dois túbulos separados, duas sensilas finas de guarda e pequena sensila ventral; antenômero IV com vesícula apical trilobada, uma micro sensila arredondada alojada em fosseta e cinco sensilas longas e pouco espessas entre cerdas muito longas e finas; face ventral e apical do antenômero IV com várias cerdas truncadas portando pequeno filamento (Fig. 19). 
Cornéulas 7+7; órgão pós-antenal ausente, havendo em substituição pequena área de granulação mais fina (Fig. 20). Cone bucal curto e afilado; quetotaxia labral= $3 / 222$ e cerdas labiais conforme figura 21 ; maxila estiliforme com duas lamelas sendo uma das suas extremidades em crochet; mandíbula com uma série de 25 dentes aproximadamente iguais e dois basais fortes e curvos, maiores que os outros (Fig. 22).

Tibiotarsos I, II, III, com 19, 19 e 18 cerdas respectivamente; pata III com unha medindo aproximadamente $57 \mu \mathrm{m}$, com um dente interno, sem ergot distinto (Fig. 23).

Tubo ventral com $3+3$ cerdas. Tenáculo com $3+3$ dentes (Fig. 24).

Manúbrio com 22 cerdas na face dorsal e dens pequena, com $6+6$ cerdas (Fig. 25); mucro reto com extremidade ligeiramente curva (Fig. 26); relação de comprimento dens:mucro $=1,0: 0,95$.

Placa genital do macho com cerca de 31 cerdas dispostas em dois círculos e aspecto ventral das valvas conforme figura 27; placa genital da fêmea com cerca de 15 cerdas dispostas em semicírculo (Fig. 28).

Material examinado. BRAsil, São Paulo: Ubatuba (Distrito de Picinguaba, Parque Estadual da Serra do Mar), 2 machos em lâminas 367 CM/MNRJ, 16-VII1991, R. Machado leg.; 2 machos e 1 fêmea em lâminas 374 CM/MNRJ, 22-VIII-91, R. Machado leg.; 1 espécime em álcool 70 \% 374 CM/MNRJ, 22-VIII-1991, R. Machado leg.; 1 fêmea em lâmina 378 CM/MNRJ, 22-VIII-1991, R. Machado leg.; Rio de Janeiro: Valença (Santuário da Concórdia), 1 fêmea em lâmina 736 CM/MNRJ, 10-VI-1997, E. Calil leg. .

Discussão. Arlesia proxima foi descrita brevemente a partir de material procedente de Mangaratiba, Estado do Rio de Janeiro, e só recentemente foi encontrada no Parque Estadual da Serra do Mar, Núcleo Picinguaba, Estado de São Paulo. Embora os exemplares correspondam a diagnose dada por ARLÉ (1939: 69-70; figs 11, 12), acredita-se ser conveniente apresentar uma recaracterização detalhada com base nos exemplares coletados em Picinguaba.

Em termos de padrão de coloração, esses espécimens enquadram-se perfeitamente à descrição original, no entanto, foram observadas algumas variações neste tocante. Descrevendo a coloração, ARLÉ (1939) cita amarelo-alaranjado para as manchas arredondadas dorsolaterais no tergito torácico II. Entretanto, nos espécimens aqui estudados, essas manchas também apresentam cor esbranquiçada. Além disto, encontrou-se recentemente um exemplar procedente do Santuário da Concórdia, Valença, Rio de Janeiro, com coloração uniformemente azulada, portando os dois últimos antenômeros esbranquiçados. No que se refere ao habitus, A. proxima apresenta formações paratergais bastante salientes e assimétricas o que lhe confere um aspecto um tanto deformado. Com relação à morfologia da mandíbula, observou-se que a ilustração feita por ARLÉ (1939) para A. proxima mostra a série de dentes entre o apical e os dois basais formada por dentes maiores e menores, dispostos alternadamente. Nos espécimens aqui estudados, a referida série é composta por cerca de 25 dentes aproximadamente do mesmo tamanho, e a maxila é do tipo estiliforme, com uma das extremidades em crochet. Verificou-se ainda, na face ventral do antenômero IV, a presença de cerdas truncadas com pequeno filamento. 
AGRADECIMENTOS. Aos colegas Dr. J.G. Palacios-Vargas da Universidade Nacional Autônoma do México, e Dr. P. Bellinger da California State University, pela leitura crítica e valiosas sugestões. Ao colega do Museu Nacional, Dr. Alcimar do Lago Carvalho pelas fotografias aqui apresentadas e ao Dr. Roberto Machado, do Instituto Oswaldo Cruz, pelo material coletado em Picinguaba. Agradecemos ainda aos dois revisores anônimos da Revista Brasileira de Zoologia pela leitura criteriosa e sugestões ao manuscrito.

\section{REFERÊNCIAS BIBLIOGRÁFICAS}

ARLÉ, R. 1939. Novas espécies de Pseudachorutini (Collembola) do Rio de Janeiro e arredores. Bol. Biol. (N.S.) 4 (1): 67-72.

ARLÉ, R. \& E. RUFINO. 1976. Contribuição ao conhecimento dos Pseudachorutinae da Amazônia (Collembola). Acta Amazonica 6 (1): 99-107.

ICZN. 1985. International Code for Zoological Nomenclature. Third Edition adopted by the $20^{\text {th }}$ General Assembly of the International Union of Biological Sciences. London, International Trust for Zoological Nomenclature \& British Museum (Natural History), XX+338p.

DENIS, J.R. 1931. Contributto alla conoscenza del "microgenton" di Costa Rica, II. Collemboles de Costa Rica avec une contribution au species de l'ordre. Boll. Lab. Entomol. Agr. Portici 25: 69-170.

HANDSCHIN, E. 1942. Materialien zur Revision der Collembolen die gattung Ceratrimeria C.B. sensu Womersley. Ver. Naturz. Ges., Basle, 53: 265-284.

Massoud, Z. 1963. Les Collemboles Poduromorphes du Surinanam. Stud. Fauna Surinam \& others Guyanas 6: 43-51.

- 1967. Monographie des Neanuridae, Collemboles Poduromorphes a pièces buccales modifiées. Biol. Amer. Austr. CNRS 3: 1-399.

STACH, J. 1949. The Apterygotan fauna of Poland in relation to the world fauna of this group of insects: families Anuridae and Pseudachorutidae. Acta. Mon. Musei. Hist. Nat. Poland: 1-122.

Thibaud, J.M. \& Z. MAssoud. 1983. Les Collemboles des Petites Antilles III.Neanuridae (Pseudachorutinae). Rev. Ecol. Biol. Sol. 20 (1): 111-129.

Recebido em 06.VIII.1998; aceito em 20.X.1999. 